\title{
New model of lumbar spine biomechanics with the use of simulation analysis
}

\author{
Łukasz Kubaszewski ${ }^{1}$, Mikołaj Dąbrowski ${ }^{1}$, Krzysztof Talaśka ${ }^{2}$, and Dominik Wilczyński ${ }^{2,}{ }^{*}$ \\ ${ }^{1}$ Poznań University of Medical Sciences, Department of Spondyloorthopedics and Biomechanics of \\ the Spine, Wiktor Dega Orthopedic - Rehabilitation Clinical Hospital, 28 Czerwca 1956 r. Str. \\ 135/147, 61-545 Poznań, Poland \\ ${ }^{2}$ Poznan University of Technology, Chair of Basics of Machine Design, Piotrowo Str. 3, Poland
}

\begin{abstract}
The existing biomechanical models of the lumbo-sacral spine do not explain the role of the dorsal extensor muscle and fascia. The study attempts to explain the action of paraspinal muscles based on the mechanism of contraction and "hydro skeleton". It was assumed that the muscle contracting produces hydrostatic pressure and in this way is able to resist and transfer loads. In this mechanism, inside ventricular pressure can modify the load transfer through the spine. To confirm the hypothesis discussed above, a simplified simulation model of the lumbar spine was built. For this purpose, scientific software named ABAQUS, using finite element methods was used. The model of the spine was mainly a kinematic model aimed at reflecting the impact of the spinal muscle function on lumbar lordosis.
\end{abstract}

Keywords: lumbar spine, model of lumbar spine, lumbar lordosis, biomechanics, simulation

\section{Introduction}

The causes of degenerative spine conditions are not fully recognized and the treatment approach is symptomatic. The evaluation of spine compensation determines the treatment approach for persons with degenerative spine conditions. The sagittal vertical axis compensation (SVA) accounts for anthropometric measurements of the position of the pelvis (pelvic incidence and pelvic inclination, sacral slope). However, the dimensions of the spinal curves do not affect the presence of degenerative changes in the lumbar section of the spine, and in the advanced stage thereof, there is a decrease lumbar lordosis $[1,2]$. The changes usually begins in the area of the intervertebral disc and consequently leads to the reduction of the intervertebral space which leads to a higher stress at the posterior spinal column [3]. Such biomechanics lead to altering the length of the spinal columns - a relative lengthening of the posterior spinal columns [4]. The degenerative changes and a decrease of

\footnotetext{
* Corresponding author: dominik.wilczynski@put.poznan.pl

Reviewers: Marian Dudziak, Mariana Pajtášová
} 
the lordosis cause the biomechanical stability of the lumbar section of the spine to be affected to a greater degree by the supporting functions of the anterior column.

The compensation analysis of the spinal region should not only account for the elements subject to radiological imaging, but also the spatial arrangement and the muscles stabilizing the spinal column together with the ligaments and fascia. The proper configuration of the lordosis curvature lends itself to interpreting the biomechanics of the lumbar section of the spine as similar to a suspension bridge, this would account for the high flexibility of the entire system when transferring significant loads [5].

\section{Functions of muscles in the spine}

It was demonstrated that the tension of the abdominal muscles and increased pressure in the abdomen causes reduced load in the lumbar section of the spine (in particular the mobile spinal units in the middle) [6]. This attests to the additional support function of the muscles - not only the stabilizing function. A similar function may be exhibited by the spinal erector encased in the space limited by the spinous processes as well as the lamina of the thoracolumbar fascia. According to the pneumatic principle of muscle contraction, this leads to its shortening at the same time increasing the diameter and increasing the pressure (within the limited volume of the fascial and osseous enclosure) and generating the supporting forces [5].

Together with the degenerative changes in the spine, we observe the atrophy of the spinal erector in the lower section lumbar spine. This may be related to muscle atrophy caused by unused or damaged medial branches innervating the muscle [7]. The decrease in lumbar lordosis as well as the shortening of both spinal columns in the course of the progression of degenerative changes in the spine may disturb its function by drawing closer the appendices and limiting the motion range (decrease of shrink ability). The consequence of the above is the atrophy of muscle tissue and the loss of its stabilizing function as well as, supposedly, the supporting function [5].

\section{The simulation model to confirm the formulated hypothesis}

In order to confirm the hypothesis as discussed above, a simplified simulation model of the lumbar section of the spine was built. To this end, a commercial scientific software ABAQUS was utilized, using finite element method. The spine model was primarily kinematic in order to reflect the influence of the spinal muscle function on the correct curvature of lumbar lordosis.

Fig. 1 presents a general view of the lumbar section model of the spine with indication of its components. The simplified model consists of five vertebrae corresponding anatomically to the lumbar vertebrae L1 to L5 (Fig. 1). Each vertebra is geometrically identical. The spatial placement maintains the geometric parameters characterizing the lumbar section of the spine [8]. The intervertebral joints were represented as connecting arcs characterized by the degrees of freedom of motion identical to actual joints (Fig. 1). The components joining the muscle with the vertebrae are rigid beams, these may be considered as a certain method of connecting the muscle at boundary points (viewed along the longer horizontal edge of the vertebra, the axis $z$ as on Fig. 1), in the upper, medium and lower part of the vertebra (viewed along the vertical edge of the vertebra corresponding to the longer axis of the human body, the axis $y$ as on Fig. 1).

The ligament connecting the vertebra from the side of the thoracic cage is represented as springs with set rigidity (Fig. 1). This is to represent its stabilizing function. The intervertebral discs are represented by springs with set rigidity (Fig. 1). The load assigned 
to the upper surface of the vertebra L1 is represented by point-applied force with value approx. $F=370 \mathrm{~N}=\sim 37 \mathrm{~kg}$ [8]. The pressure increasing the muscle volume is $p=150 \mathrm{kPa}$ (Fig. 1). Such value was assumed based on the deformation characteristic of the material used to model the muscle. The aim was to obtain visible deformation.

The parameterized model consists of 290162 finite elements, including 289190 linear components type Hexa C3D8R and 972 non-linear components type S4R. The material representing the vertebra has the following parameters: Young's modulus $E=12 \mathrm{GPa}$, density $\rho=2000 \mathrm{~kg} / \mathrm{m}^{3}[9-13]$.

The muscle was modeled using a material with characteristic of an elastomer where: assumed Young's modulus value is $E=0.1 \mathrm{GPa}$, density $\varrho=1300 \mathrm{~kg} / \mathrm{m}^{3}$, side thickness of the muscle model $g=1 \mathrm{~mm}$ [14]. The intervertebral joints were modeled using a material with characteristics of the ABS (Acrylonitrile butadiene styrene) polymer: Young's modulus $E=1.1 \mathrm{GPa}$, density $\rho=1200 \mathrm{~kg} / \mathrm{m}^{3}$, side thickness of the joint model $g=1.3 \mathrm{~mm}$ [14].

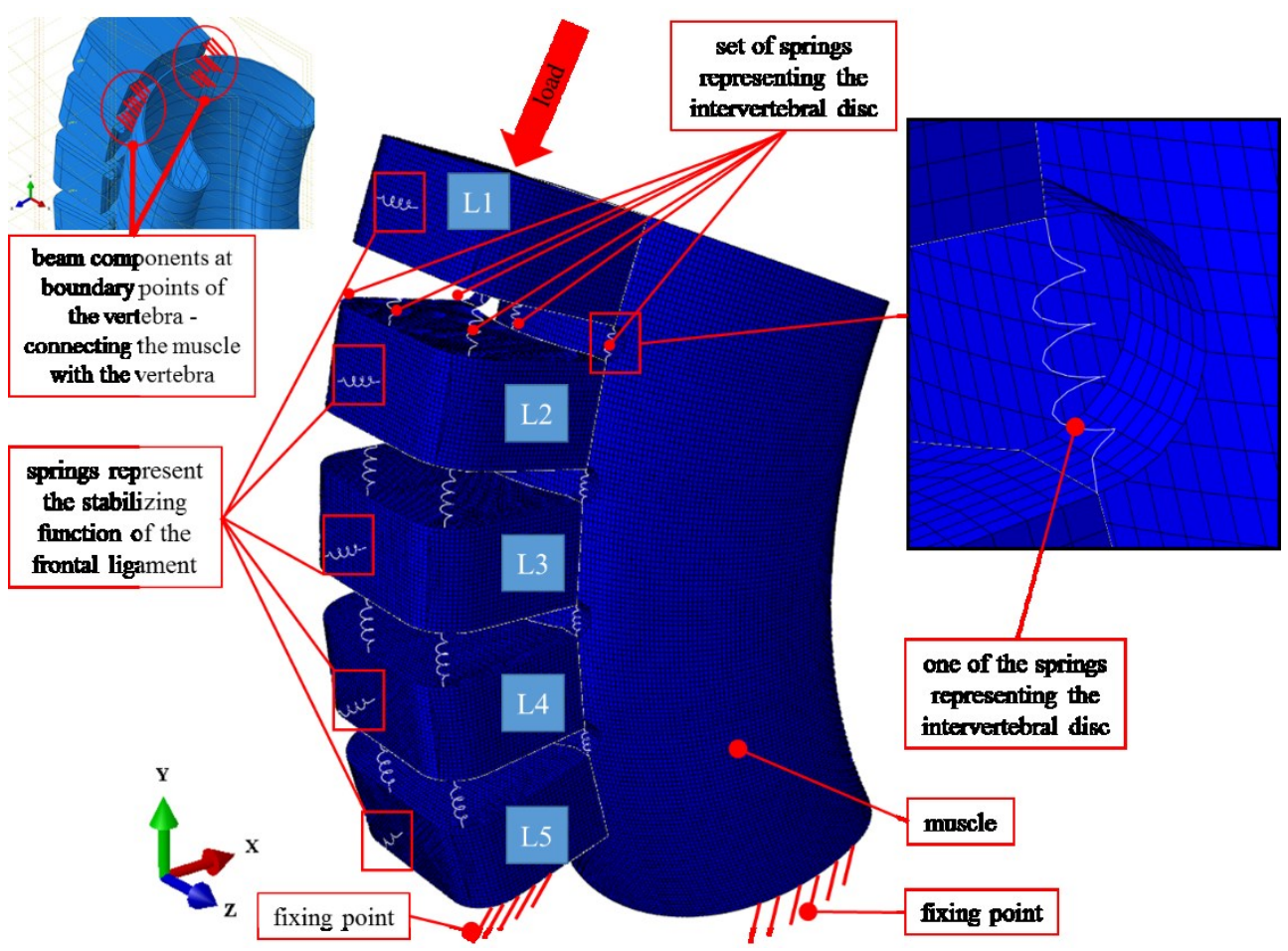

Fig. 1. General view of the simulation model of the lumbar section of the spine - view from the side of the vertebrae

Fig. 2 shows a general view of the lumbar section of the spine model viewed from the side of the muscle in order to present its transverse cross-section. 


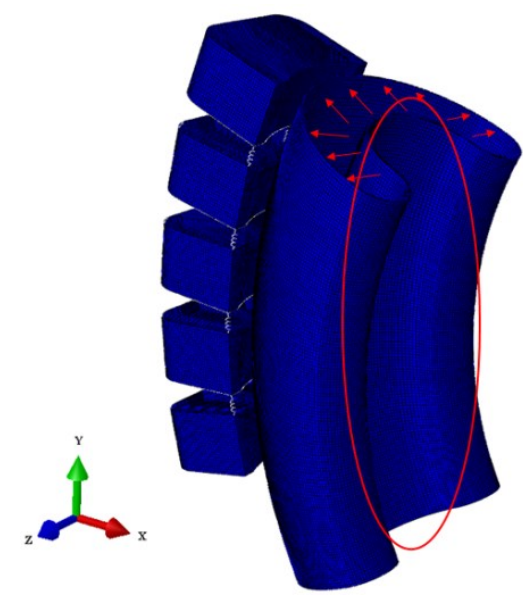

Fig. 2. General view of the simulation model of the lumbar section of the spine - view from the side of the muscle

The assumed boundary conditions include limiting the range of motion freedom from the lower and upper side of the spinal muscle in form of a translational motion along the axis $\mathrm{z}$, rotational motion along axes $\mathrm{x}$ and $\mathrm{y}$, as well as removal of all degrees of freedom of the lateral, concave side of the spinal muscle (the area indicated with an ellipsis on Fig. 2), removal of all degrees of freedom of motion of the lower part of the vertebra L5 (Fig. 1) as translational motion along axes $\mathrm{y}$ and $\mathrm{z}$, as well as rotational motion along axes $\mathrm{x}$ and $\mathrm{y}$. Furthermore, for vertebra L1 to L4 the freedom of motion was removed to prevent translational motion along axis $\mathrm{z}$ as well as rotational motion along axes $x$ and $y$. Load was assigned to the upper surface of the vertebra L1 in form of a point applied force with value equal to $F=370 \mathrm{~N}$, which is to represent the weight load of the upper human body (Fig. 1) [8]. The result of the increase of muscle volume was represented by acting on its internal sides with pressure (indicated by arrows, Fig. 2) with value equal to $p=150 \mathrm{kPa}$, which is sufficient value to achieve an increase in the volume of the spinal muscle for the assumed material characteristics of the elastomer employed in the simulation. The effect of the decrease of volume of the spinal muscle (muscle atrophy) was achieved by applying a negative pressure to its internal sides with value $p=-150 \mathrm{kPa}$ (as in the previous case, this is the sufficient value to achieve a decrease in the volume of spinal muscle for the assumed material characteristics of the elastomer employed in the simulation).

The simulation consists of three steps occurring in a sequence. Each step takes place in the course of one second. First step load equal to the force of gravity is applied, the second step adds point applied force at the upper surface of the vertebra L1, $F=370 \mathrm{~N}$ and in the third step, the pressure (negative pressure) is applied to increase (decrease) the volume of the muscle (Figs. 4 and 5). Fig. 3 indicates the displacement of the selected L3 vertebra along the axis $x$, which occurs during every step of the simulation as described above. 


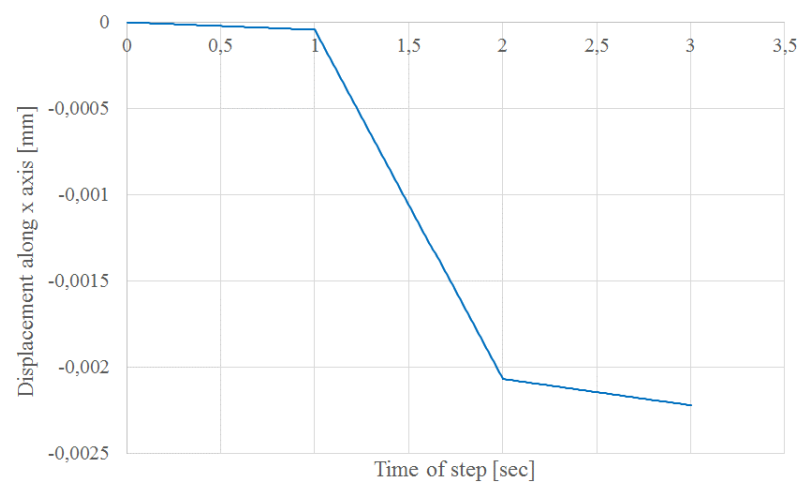

Fig. 3. Displacement of vertebra L3 along axis $\mathrm{x} w$ during each temporal step of the simulation

According to Fig. 3, the application of gravity in step one affects the displacement of the vertebra. In step two, the displacement is affected simultaneously by gravity and load applied to vertebra L1 (Fig.1). The displacement further increases in the last step, caused by the functioning of the muscle caused by the increase in its volume. Negative displacement value denotes the motion of the vertebra in the direction opposite to the direction of axis $\mathrm{x}$. One needs to point out that the order of magnitude of the displacement is not relevant, because it is affected by e.g. the value of pressure acting on the inside of the muscle model and the rigidity of springs modeling the front fixture or intervertebral discs. The aim of the simulation is to determine the effect of the function of the spinal muscles on the curvature of the lumbar lordosis.

Fig. 4 shows the results of the simulation of the proper functioning of the spinal muscle which helps to achieve the correct lumbar lordosis curvature.

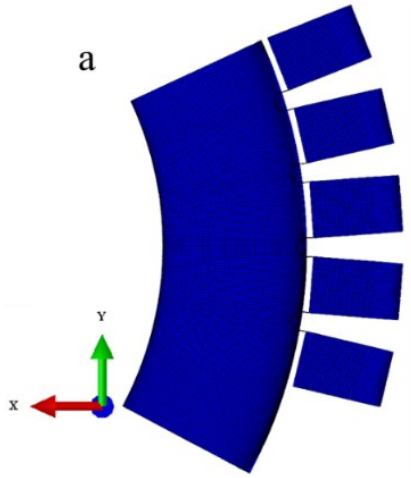

b

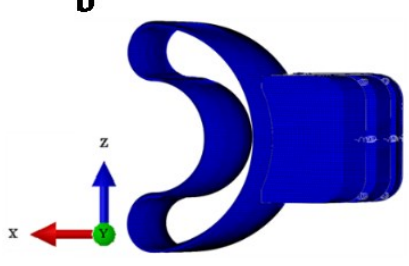

c

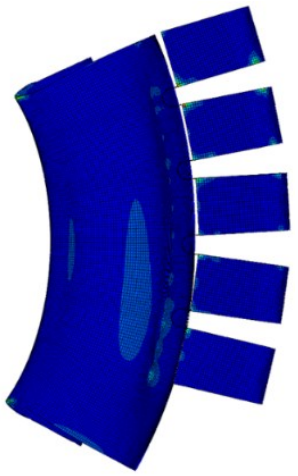

d

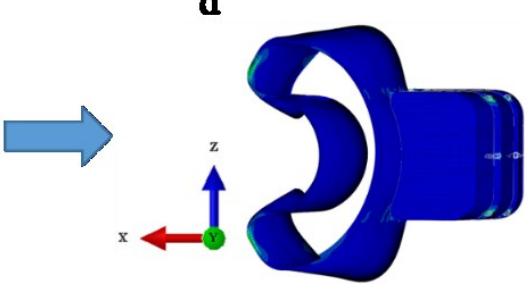

Fig. 4. Visualization of the acting of the spinal muscle on the vertebra as a result of the increase of its volume (correct function of the muscle): $a$ and $b$, two views before increasing the muscle volume, $c$ and $\mathrm{d}$, two views after increasing the muscle volume 
The two views shown at Fig. $4 \mathrm{a}$ and $4 \mathrm{~b}$ represent the state of the model before starting the simulation. The subsequent views on Fig. $4 \mathrm{c}$ and $4 \mathrm{~d}$ represent the end result of the simulation.

According to the above, the increase in volume of the spinal muscle is visible, causing the vertebrae to be pushed away in the direction opposite to the direction of axis $x$ (fig. $4 \mathrm{a}$ and $4 \mathrm{~b}$ ), which means that proper curvature of lumbar lordosis can be maintained. Viewed from along the axis $y$, the distance between them is increased. Fig. 5 shows the results of simulating incorrect functioning of the spinal muscle (muscular atrophy), which contributes to the diminishing of the lordosis curvature.
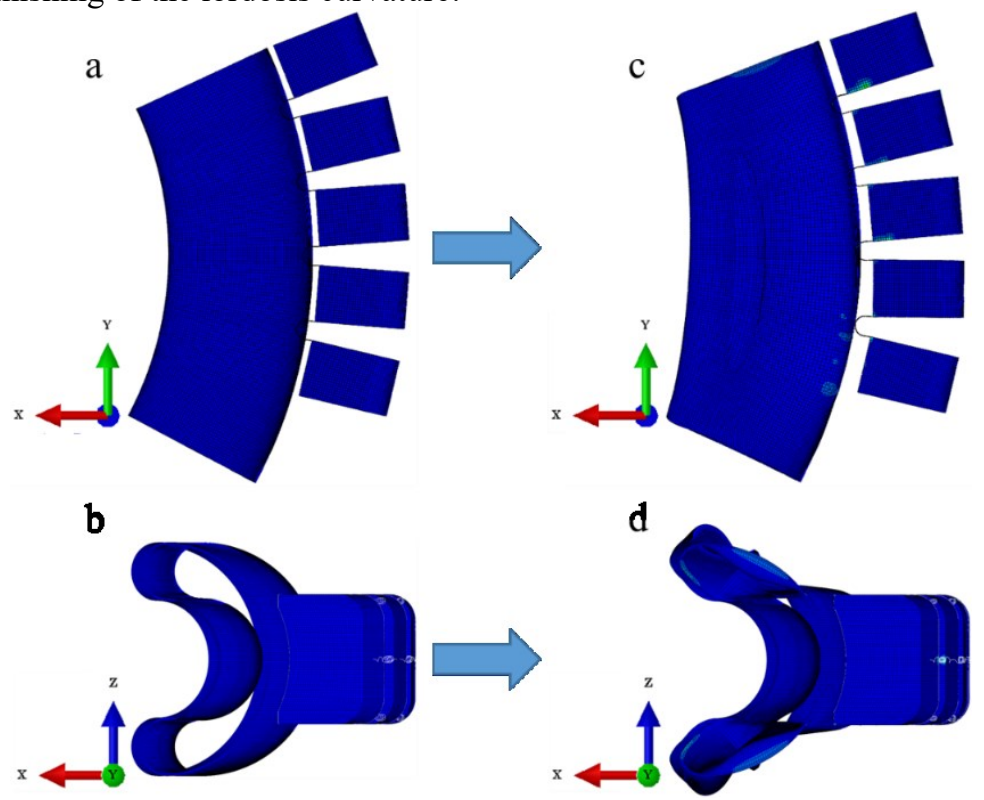

Fig. 5. Visualization of the simulated action of the spinal muscle on the vertebra resulting from the decrease of its volume (muscular atrophy): $a$ and $b$, two views representing the stages before the decrease of the muscle volume, $\mathrm{c}$ and $\mathrm{d}$ are two views after the decrease in muscle volume

Two views provided on Fig. 5a and $5 \mathrm{~b}$ represent the state of the model before the beginning of the simulation. The subsequent views on Fig. $5 \mathrm{c}$ and $5 \mathrm{~d}$ represent the final result of the simulation.

According to the above, there is a noticeable decrease in spinal muscle volume (muscular atrophy - improper functioning), which causes a retraction of the vertebrae (withdrawing) in the direction corresponding to the direction of the axis $x$ (Fig. 5). This causes proper spinal lordosis curvature to disappear. When viewed from the axis $y$, we see a decrease of the distance between the vertebrae.

As a result of the carried out simulations (Figs. 4 and 5), the characteristics for the function of value of the reaction force in time (Fig. 6) were obtained, the force acting on vertebra L5 as an effect of propagation of the load on vertebra L1 (Fig. 1). The determined value constitutes a component of the reaction force acting along the axis y (Fig. 1), which affects the intervertebral discs. 


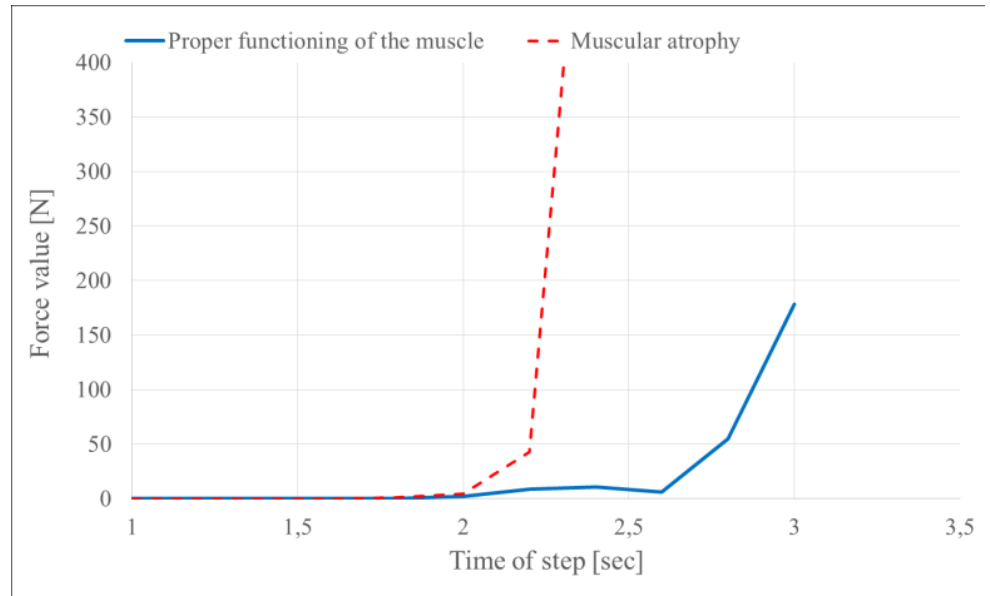

Fig. 6. Function of reaction force value acting along axis y for vertebra L5

The characteristic indicated with the blue line (solid line) (Fig. 6) indicates correct functioning of the spinal muscle, which in reality and in the simulated environment corresponds to the increase of its volume (in reality: the growth of a healthy muscle tissue which is functioning properly). The correct functioning of the muscle causes a decrease in the reaction force acting along axis $y$ (Fig. 1) to the value $F \approx 180 \mathrm{~N} \approx 18 \mathrm{~kg}$, in reference to the load value affected on vertebra $\mathrm{L} 1, F=370 \mathrm{~N} \approx 37 \mathrm{~kg}$. With muscular atrophy, the constituent reaction force acting along the axis y increases rapidly (the characteristic indicated with the red line - broken line, Fig. 6). This may indicate excessive load on the intravascular discs causing pathological changes in them as well as for the entire system of the lumbar spine [15].

The conducted analyses are the basis for confirming the hypothesis formulated earlier, regarding the additional support function of the spinal muscles. The further course of study is to perform a series of numerical simulations on a more sophisticated model of the spinal column. The results obtained to date confirm that the hypothesis is true and give grounds to carry out further research in this direction.

\section{Summary}

The study presents the results of the numerical simulation performed in the ABAQUS system regarding the impact of the action of the spinal muscle on the vertebra located on the lumbar section of the spinal column. The results obtained allow for the preliminary confirmation of the hypothesis. Two cases were simulated: correct functioning of the muscle and its atrophy. In the first scenario, the beneficial effect of the muscle functioning on the lumbar curvature of the spine was determined. The distance between the vertebrae is increased which serves to reduce the negative action of the loads affecting the intervertebral disc. With muscular atrophy, the lumbar lordosis decreases and supports the development of the degenerative disease.

The obtained results allow to make a decision regarding further course of study in this direction which entails building another, more sophisticated simulation model. The subsequent results that confirm the correctness of the hypothesis allow to formulate a new approach in treating the conditions of the lumbar spine. 


\section{References}

1. R.P. Jackson, A.C. McManus, Radiographic analysis of sagittal plane alignment and balance in standing volunteers and patients with low back pain matched for age, sex, and size. A prospective controlled clinical study. Spine, 19, 1611-1618 (1994)

2. C. Barrey, J. Jund, O. Noseda et al., Sagittal balance of the pelvis-spine complex and lumbar degenerative diseases. A comparative study about 85 cases. Eur. Spine J, 16, 1459-1467 (2007)

3. A. Fujiwara, K. Tamai, M. Yamato et al., The relationship between facet joint osteoarthritis and disc degeneration of the lumbar spine: an MRI study. Eur. Spine J., 8, 396-401 (1999)

4. R. Maes, W.B. Morrison, L. Parker et al., Lumbar interspinous bursitis (Baastrup disease) in a symptomatic population: prevalence on magnetic resonance imaging. Spine, 33, E211-215 (2008)

5. Ł. Kubaszewski, T. Kotwicki, A. Nowakowski, P. Lisiński, J. Kaczmarczyk, Nowe spojrzenie na zagadnienie kompensacji kręgostupa $w$ przebiegu choroby zwyrodnieniowej. Chir. Narządów Ruchu Ortop. Pol, 80(6), 207-213, (2015)

6. F.H. Willard, A. Vleeming, M.D. Schuenke et al., The thoracolumbar fascia: anatomy, function and clinical considerations. J Anat., 221, 507-536, (2012)

7. P. Hodges, A.K. Holm, T. Hansson et al., Rapid atrophy of the lumbar multifidus follows experimental disc or nerve root injury. Spine (Phila $\mathrm{Pa}$ 1976), 31, 2926-2933 (2006)

8. A.I. Kapandji, The Physiology of the joints, the spinal column, pelvic girdle and head, Vol. 3, Edition 6

9. A. Rohlmann, T. Zander, H. Schmidt, H.J. Wilke, G. Bergmann, Analysis of the influence of disc degeneration on the mechanical behaviour of a lumbar motion segment using the finite element method, Journal of Biomechanics Vol. 39 Issue (13), p. 2484-2490 (2006)

10. H. Schmidt, A. Kettler, A. Rohlmann, L. Claes, H.J. Wilke, The risk of disc prolapses with complex loading in different degrees of disc degeneration-a finite element analysis, Clinical Biomechanics (Bristol, Avon) Vol. 22 Issue (9) p. 988-998 (2007)

11. H. Schmidt, F. Heuer, H.J. Wilke, Dependency of disc degeneration on shear and tensile strains between annular fiber layers for complex loads, Medical Engineering and Physics Vol. 31 Issue (6), p. 642-649 (2009)

12. L.M. Ruberte, R.N. Natarajan, G.B. Andersson, Influence of single-level lumbar degenerative disc disease on the behaviour of the adjacent segments - a finite element model study, Journal of Biomechanics Vol. 42 Issue (3), p. 341-348 (2009)

13. V. K. Goel, B.T. Monroe, L.G. Gilbertson, P. Brinckmann, Interlaminar shear stresses and laminae separation in a disc. Finite element analysis of the L3-L4 motion segment subjected to axial compressive loads, Spine (Phila Pa 1976) Vol. 20 Issue (6), p. 689 698 (1995)

14. M. Ashby, H. Shercliff, D. Cebon, Materials engineering, science, processing and design Edition 2, Elsevier (2010)

15. D. Hong-gen, L. Sheng-hui, J. Zhong, H. Huan-ming, N. Xi-tao, J. Neng-yi, P. Jian-wei, H. Qin, W. Hui, Biomechanical analysis of press-extension technique on degenerative lumbar with disc herniation and staggered facet joint, Saudi Pharmaceutical Journal 24, p. 305-311 (2016) 